\title{
$p$-SOLVABLE GROUPS WITH FEW AUTOMORPHISM CLASSES OF SUBGROUPS OF ORDER $p$
}

\author{
FLETCHER GROSS ${ }^{1}$
}

\begin{abstract}
This paper investigates the relationship between the $p$-length, $l_{p}(G)$, of the finite $p$-solvable group $G$ and the number, $a_{p}(G)$, of orbits in which the subgroups of order $p$ are permuted by the automorphism group of $G$. If $p>2$ and $a_{p}(G) \leqq 2$, it is shown that $l_{p}(G) \leqq a_{p}(G)$. If $p=2$ and $a_{2}(G)=1$, it is proved that either $l_{p}(G) \leqq a_{p}(G)$ or $G / O_{2}(G)$ is a specific group of order 48 .
\end{abstract}

1. Introduction. If $p$ is a prime, $G$ is a finite $p$-solvable group, and the automorphisms of $G$ permute the subgroups of $G$ of order $p$ in exactly $a_{p}(G)$ orbits (this defines $a_{p}(G)$ ), what, if any, is the relationship between $a_{p}(G)$ and $l_{p}(G)$, the $p$-length of $G$ ? If $p$ is odd and $a_{p}(G)$ $=1$, then Shult [6] proved that $l_{p}(G)=1$. Thompson [7, Lemma 5.40] showed that $l_{2}(G)=1$ if a Sylow 2-group of $G$ contains more than one involution and the inner automorphisms of $G$ transitively permute the involutions of $G$.

The main results of the present paper are:

(1) If $G$ is a finite $p$-solvable group, $p>2$, and $a_{p}(G)=2$, then $l_{p}(G) \leqq 2$;

(2) If $G$ is a finite solvable group and $a_{2}(G)=1$, then either $l_{2}(G)=1$ or $G / O_{2^{\prime}}(G)$ is a specific group of order 48 and $l_{2}(G)=2$.

The upper bound in (1) is best possible as is shown by the following example. Let $p$ be a prime, let $V$ be the additive group of the field $\mathrm{GF}\left(p^{p}\right)$, and let $\lambda$ be a generator of the multiplicative group of $\mathrm{GF}\left(p^{p}\right)$. Define the automorphisms $A$ and $B$ of $V$ by $x A=\lambda x$ and $x B=x^{p}$ for $x \in V . A$ has order $p^{p}-1, B$ has order $p$, and $B^{-1} A B=A^{p}$. If $G$ is the semidirect product of $V$ and the group generated by $A$ and $B$, then $G$ is solvable, $a_{p}(G)=2$ (in fact the inner automorphisms of $G$ permute the subgroups of order $p$ in 2 orbits), and $l_{p}(G)=2$.

2. Notation and preliminary results. The concepts of $p$-solvability and $p$-length are defined in [4]. It should be noted that a 2 -solvable

Presented to the Society, April 24, 1971 under the title Finite groups with a small number of automorphism classes of subgroups of order p; received by the editors November 16, 1970.

AMS 1970 subject classifications. Primary 20D10, 20D45; Secondary 20D30.

Key words and phrases. p-solvable groups, p-length, automorphism classes, p-subgroups.

1 Research supported in part by NSF Grant GP-12028.

Copyright @ 1971, American Mathematical Society 
group is solvable because of the Feit-Thompson Theorem [2]. All group characters considered in this paper are over the field of complex numbers. If $\chi$ is a character of $G$, then $e_{\chi}=\left(\sum_{x \in G} \chi\left(x^{2}\right)\right) /|G|$. It is known [1, Theorem 3.5] that $e_{\chi}=1,-1$, or 0 depending on whether $\chi$ is the character of a real representation, $\chi$ is real valued, but is not the character of a real representation, or $\chi$ is not real valued, respectively. The rest of the notation used agrees with [2].

LemMa 2.1 (Тномpson [7, Lemma 5.17]). Let $G$ be a finite group, $P$ a normal p-subgroup of $G$, and $Q$ a subgroup of $G$ of order not divisible by $p$. If $[P, Q]=P \neq 1$, and $Q$ centralizes every characteristic abelian subgroup of $P$, then $P$ is a nonabelian special p-group.

Lemмa 2.2. Let $G$ be a finite group, $P$ a normal p-subgroup of $G, Q$ a subgroup of $G$ of order not divisible by $p$, and $C=C_{P}(Q)$. A ssume either $p>2$ or $P^{\prime}=1$. If every element of order $p$ in $P$ is conjugate in $P$ to an element of $C$, then $C=P$.

Proof. If $C \neq P$, then there is an integer $n$ such that $Z_{n}(P) \leqq C$, but $Z_{n+1}(P)=C$. Let $x$ be any element of order $p$ in $Z_{n+1}(P)$. Then $x^{y} \in C$ for some $y \in P$. Since $x^{y} \equiv x\left(\bmod Z_{n}(P)\right)$, it follows that $x \in C$. A theorem of Huppert [5] now implies that $Z_{n+1}(P) \leqq C$.

LEMMA 2.3. Let $G$ be the group with generators $x$ and $y$ and relations $x^{4}=y^{3}=x^{2}(x y)^{4}=1$. Then $|G|=48,|Z(G)|=2, G / Z(G)$ is isomorphic to $S_{4}, l_{2}(G)=2$, and a Sylow 2-group of $G$ is generalized quaternion.

Proof. $G=\langle x, x y\rangle$ and $x^{2}=(x y)^{4}$. Thus $x^{2} \in Z(G) . G /\left\langle x^{2}\right\rangle$ is generated by two elements $\bar{x}$ and $\bar{y}$ where $\bar{x}^{2}=\bar{y}^{3}=(\bar{x} \bar{y})^{4}=1$. It follows that $G /\left\langle x^{2}\right\rangle$ is a homomorphic image of $S_{4}$. Therefore, $|G| \leqq 48$. Now it is verified easily that the permutations

$$
(1,2,3,4)(5,6,7,8)(9,10,11,12)(13,14,15,16)
$$

and

$$
(2,5,9)(4,7,11)(6,12,13)(8,10,15)
$$

satisfy our relations and generate a group of order at least 48 . Hence $|G|=48$ and $G /\left\langle x^{2}\right\rangle$ is isomorphic to $S_{4}$. Since $Z\left(S_{4}\right)=1$, this implies that $Z(G)=\left\langle x^{2}\right\rangle$. It is possible to choose the homomorphism of $G$ onto $S_{4}$ such that $x$ is mapped onto (12) and $y$ is mapped onto (134). Then if $z$ is any involution in $G, z$ must be conjugate in $G$ to an element of either $Z(G) x$ or $Z(G)(x y)^{2}$. It now follows that $x^{2}$ is the only involution in $G$. Hence a Sylow 2-group of $G$ is generalized quaternion. 
LEMMA 2.4. Let $G$ be a finite solvable group of 2-length $>1$ such that a Sylow 2-group of $G$ has only one involution. Assume that $O_{2^{\prime}}(G)=1$. Then $l_{2}(G)=2$, a Sylow 2-group of $G$ is generalized quaternion, $|Z(G)|=2, G / Z(G)$ is isomorphic to $S_{4}$, and $G$ is isomorphic to the group with generators $x$ and $y$ and relations $x^{4}=y^{3}=x^{2}(x y)^{4}=1$.

Proof. Let $P=O_{2}(G)$. Then $C_{G}(P)=Z(P)$ [4, Lemma 1.2.3], and $G / P$ is not a 2 -group but has order divisible by 2 . Thus $G / Z(P)$ is isomorphic to a subgroup of the automorphism group of $P$. Since $P$ has only one involution, $P$ is either cyclic or generalized quaternion. It now follows that $P$ must be quaternion of order 8 (otherwise $G / Z(P)$ would have to be a 2 -group). The automorphism group of $P$ then is isomorphic to $S_{4}$ and $Z(P)=Z(G)$. Since $l_{2}(G / Z(P))=l_{2}(G)>1$, it follows that $G / Z(G)$ is isomorphic to $S_{4}$. Then $|G|=48$. It only remains to show that $G$ is generated by two elements satisfying the above relations.

Now $S_{4}$ is generated by the two permutations (12) and (134). Thus $G$ contains two elements $x$ and $y$ such that $y$ has order $3, G / Z(G)$ is generated by $x Z(G)$ and $y Z(G)$, and $x Z(G)$ and $(x y) Z(G)$ have orders 2 and 4 , respectively, in $G / Z(G)$. Since $G$ has only one element of order 2 , it follows that $G=\langle x, y\rangle$ and $x^{4}=y^{3}=x^{2}(x y)^{4}=1$.

TheOREM 2.5. Let $P$ be a 2-group of exponent 4 such that $a_{2}(P)=1$. Assume $1<r=\left|\Omega_{1}(P)\right|<|P|$. Then $\Omega_{1}(P)=D(P) \leqq Z(P), Z(P)$ is either $P$ or $\Omega_{1}(P)$, and $P^{\prime}$ is either 1 or $\Omega_{1}(P)$. If $\chi$ is an irreducible character of $P$ whose kernel does not contain $\Omega_{1}(P)$, then one of the following must be satisfied:

(a) $|P|=r^{2}$ and $e_{\chi}=0$.

(b) $P$ is a nonabelian special 2-group of order $r^{3}, \chi(1)=r, e_{\chi}=-1$, and $\left|C_{p}(x)\right|=r^{2}$ if $x$ is any element of order 4 in $P$.

Proof. Since $e(P)=4, P / \Omega_{1}(P)$ has exponent 2 and so is abelian so $P^{\prime} \leqq \Omega_{1}(P)$. Since $D(P)$ is generated by commutators and squares (which lie in $\Omega_{1}(P)$ ), $D(P) \leqq \Omega_{1}(P)$. Since the automorphisms of $P$ transitively permute the involutions in $P$, we must have $D(P)$ $=\Omega_{1}(P) \leqq Z(P)$. Now let $M=D(P)$. If $x \in M^{\sharp}$, let $m$ be the number of solutions in $P$ to $y^{2}=x . m$ is independent of $x$ and so $|P|=m(r-1)+r$. Hence $(r-1)$ divides $(|P|-1)$. Since $r$ and $|P|$ are both powers of 2, this implies that $|P|=r^{n}$ for some integer $n>1$ and then $m=\left(r^{n}-r\right) /(r-1)$. A similar argument yields that $|Z(P)|$ is a power of $r$. Now if either $|Z(P)|>r^{2}$ or $|Z(P)|=r^{2}<r^{n}$, then there are elements $x$ and $y$ in $P-M$ such that $M x \neq M y,[x, y]=1$, and $x^{2}=y^{2}$. 
But then $(x y)^{2}=1$ is contrary to $x y \notin M$. Hence either $Z(P)=M$ or $|Z(P)|=|P|=r^{2}$. Thus if $P$ is abelian, $n=2$, and we see that $P$ is the direct product of several cyclic groups of order 4 . Then, since $M$ is not in the kernel of $\chi, \chi$ cannot be real valued and so $e_{\chi}=0$.

Now suppose $P^{\prime} \neq 1$. Then $P^{\prime} \cap M \neq 1$ and so $P^{\prime} \geqq M=D(P) \geqq P^{\prime}$. Hence $P^{\prime}=M$. Let $N$ be the intersection of $M$ and the kernel of $\chi$. $N$ must have order $r / 2 . \chi\left(x^{2}\right)=\chi(1)$ if $x^{2} \in N$, and since $M / N$ $\leqq Z(P / N), \chi\left(x^{2}\right)=-\chi(1)$ if $x^{2} \notin N$. It now follows that

$$
\begin{aligned}
r^{n} e_{\chi} & =\chi(1)[m(|N|-1)+r-m(|M|-|N|)] \\
& =-\chi(1)\left(r^{n}-r^{2}\right) /(r-1) .
\end{aligned}
$$

If $n=2$, then $e_{\chi}=0$. Suppose now $n>2$. Then we must have $e_{\chi}=-1$ and $\chi(1)=r^{n-2}(r-1) /\left(r^{n-2}-1\right)$. For this to be an integer, we must have $n=3$. Then $\chi(1)=r$. Since $|P| \neq r^{2}, Z(P)=M$. $P$ now is a special nonabelian 2-group.

Finally let $x$ be an element of order 4 in $P$. Then all conjugates of $x$ are contained in $M x$. Hence $\left|P: C_{P}(x)\right| \leqq r$. If $\left|P: C_{P}(x)\right|<r$, then $P$ must have more than $r+\left(r^{3}-r\right) / r=r^{2}+r-1$ conjugacy classes. Since $P$ has exactly $r^{2}$ linear characters, this would imply that $P$ has at least $r$ irreducible characters of degree $r$. Since $r^{2}+r(r)^{2}>|P|$, this is impossible. Hence $\left|P: C_{P}(x)\right|=r$ and so $\left|C_{P}(x)\right|=r^{2}$.

\section{The main results.}

TheOREM 3.1. If $G$ is a finite p-solvable group for the odd prime $p$ and $a_{p}(G)=2$, then $l_{p}(G) \leqq 2$.

Proof. Let $G$ be a counterexample to the theorem of minimal order. Then $O_{p^{\prime}}(G)=1$. Let $A$ be the holomorph of $G$, let $S=O_{p}(G)$, and let $H$ be a Hall $p^{\prime}$-subgroup of $O_{p p^{\prime}}(G)$. The Frattini argument implies that $A=S N_{A}(H)$. Let $M=S \cap N_{A}(H)$. If $M=1$, it follows easily that any two subgroups of order $p$ in $N_{G}(H)$ are conjugate in $N_{A}(H)$. Thus $a_{p}(G / S) \leqq 1$. This implies that $l_{p}(G / S) \leqq 1[6]$. Since $l_{p}(G / S)$ $=l_{p}(G)-1$, this is a contradiction.

Hence $M \neq 1$. $M=C_{S}(H)$ and so, from Lemma 2.2, not every element of order $p$ in $S$ can be conjugate in $S$ to an element of $M$. Since $A=S N_{A}(H)=S N_{A}(M)$, it follows that there is an element of order $p$ in $S$ which is not conjugate in $A$ to any element of $M$. Since $a_{p}(G)=2$, this implies that $S$ contains all elements of order $p$ in $G$ and that any two subgroups of order $p$ in $M$ are conjugate in $A$. If it were true that all subgroups of order $p$ in $M$ were conjugate in $N_{A}(H)$, then it would follow that $a_{p}\left(N_{G}(H)\right)=1$. But this would imply that $l_{p}(G)=l_{p}(G / S)+1 \leqq 2$. 
Thus there are two subgroups $P_{1}$ and $P_{2}$ of order $p$ in $M$ that are not conjugate in $N_{A}(H)$. We must have $P_{2}=P_{1}^{x y}$ for some $x \in N_{A}(H)$ and some $y \in S$. Let $z$ be a generator of $P_{1}$. Since $P_{2} \neq P_{1}^{x}$, we obtain $1 \neq\left[z^{x}, y\right] \in M \cap\left[\Omega_{1}(M), S\right]$. But if $P$ is a subgroup of order $p$ contained in $M \cap\left[\Omega_{1}(M), S\right]$, then every subgroup of order $p$ in $M$ is conjugate in $A$ to $P$. Since $\left[\Omega_{1}(M), S\right] \triangleleft S N_{A}(M)=A$, it follows that $\left[\Omega_{1}(M), S\right] \geqq \Omega_{1}(M)$. Since $S$ is nilpotent, we have a contradiction and the theorem is proved.

THEOREM 3.2. Let $G$ be a finite solvable group such that $a_{2}(G)=1$. Then either $l_{2}(G) \leqq 1$ or $l_{2}(G)=2$ and $G / O_{2^{\prime}}(G)$ is a group of order 48 which is isomorphic to the group with generators $x$ and $y$ and relations $x^{4}=y^{3}=x^{2}(x y)^{4}=1$.

Proof. Suppose $G$ is a counterexample to the theorem of minimal order. Because of Lemma 2.4, a Sylow 2-group of $G$ must have more than one involution. It now follows that $O_{2^{\prime}}(G)=1$ and $G=O_{22^{\prime}}(G)$. Now let $A$ be the holomorph of $G$. Then $G \triangleleft A$ and all involutions of $G$ are conjugate in $A$. Let $S=O_{2}(G), M=\Omega_{1}(Z(S)), Q=$ a Hall $2^{\prime}$ subgroup of $G, P=$ a Sylow 2-subgroup of $N_{G}(Q)$, and $N=N_{A}(Q)$ $\cap N_{\Delta}(P)$. Then the Fitting argument yields $G=S Q P, A=N G$, $N_{G}(Q)=P Q$, and $N_{A}(Q)=N P Q$.

Since $l_{2}(G)>1$, we must have $G \neq S Q$. Since $M \triangleleft A, M$ must contain all involutions in $G$. Now $1 \neq M \cap N_{A}(Q) \triangleleft A$ since $A=N_{A}(Q) S$ and $M \leqq Z(S)$. It follows that $M \leqq N_{A}(Q)$. This implies that $M \leqq P$ and $[M, Q]=1$. Now $M \cap Z(P) \neq 1$ and $M \cap Z(P) \triangleleft S Q N_{A}(P)=A$. Thus $M \leqq Z(P)$. It follows that $M \leqq Z(G)$ and so conjugation by elements of $N$ transitively permutes the nonidentity elements of $M$.

Suppose $K$ is a proper subgroup of $Q$ which is normalized by $P N$. Then $S K P$ satisfies the hypothesis of the theorem and has smaller order than $G$. Thus, $l_{2}(S K P)=1$ which implies that $[P, K]=1$. It now follows (see, for example, [3, Lemma 2.4]) that $Q$ is a special $q$-group for some odd prime $q, N P$ transforms $Q / Q^{\prime}$ irreducibly, and $C_{Q}(P)=Q^{\prime}$.

Since $Q$ centralizes $M, Q$ must centralize any abelian 2-subgroup of $G$ which is normalized by $Q$. Suppose now $L=[S, Q] .1 \neq L \leqq Q$ and $L \triangleleft A$. Thus $L$ must contain $M$. Now $L=[L, Q]$ and $Q$ centralizes any characteristic abelian subgroup of $L$. Thus, by Lemma $2.1, L$ is a nonabelian special 2-group. It follows that $L^{\prime}=M$. Since $L / M=\left(C_{L}(Q) / M\right) \times([L / M, Q])$, we have $C_{L}(Q)=M$. Suppose now $L<S$. Then $S$ contains a subgroup $R$ such that $L<R$ and $R / L$ is a minimal normal subgroup of $A / L$. Since $[R, Q]=L, R=L C_{R}(Q)$ and so $C_{R}(Q)$ is not contained in $L$. Now let $T / M=C_{L / M}(R)$. Then $T \triangleleft A$ 
and $T>M$. Since $\left[C_{R}(Q), T, Q\right] \leqq[M, Q]=1$ and $\left[Q, C_{R}(Q), T\right]=1$, the three subgroups lemma implies that $\left[T, Q, C_{R}(Q)\right]=1$. But $T=C_{T}(Q)[T, Q]=M[T, Q]$. It now follows that $\left[T, C_{R}(Q)\right]=1$. Let $x \in C_{R}(Q)-L$. Then $x^{2} \neq 1$, but $C_{R}(Q) /\left(L \cap C_{R}(Q)\right)$ is isomorphic to the elementary abelian group $R / L$. Hence $x^{2} \in L \cap C_{R}(Q)=C_{L}(Q)$ $=M$. Now $\left\{y^{2} \mid y \in T-M\right\}$ is a subset of $M^{\#}$ normalized by $A$. Hence there is a $y$ in $T-M$ such that $y^{2}=x^{2}$. Since $\left[C_{R}(Q), T\right]=1,(x y)^{2}$ $=x^{2} y^{2}=1$ contrary to $x y \notin M$. Thus $L=S$. Therefore $S$ is a nonabelian special 2-group, $S^{\prime}=M,[S, Q]=S$, and $C_{S}(Q)=M$. Since $S \cap P$ $\leqq C_{S}(Q)$, we have $S \cap P=M$.

Next let $r=|M| . r>2$ and, by Theorem 2.5, $|S|=r^{2}$ or $r^{3}$. If $|S|=r^{2}$ and $y \in M^{\sharp}$, then the solutions in $S$ to $x^{2}=y$ will constitute a single coset of $M$. Since $[M, Q]=1$, this would imply that $[S / M, Q]$ $=1$. Since this contradicts $[S, Q]=S$, we must have $|S|=r^{3}$. Then, by Theorem $2.5,\left|C_{S}(x)\right|=r^{2}$ for all $x \in S-M$.

If $M<H<P$ and $N$ normalizes $H$, then $S Q H$ is a counterexample to the theorem of smaller order than $G$. Hence $G / S Q$ is a minimal normal subgroup of $A / S Q$. Therefore $P / M$ is elementary abelian. The elements of $N$ induce automorphisms of $P$ which transitively permute the involutions in $P$. Hence $|P|=r^{n}$ where $n=2$ or 3 .

Now let $C / M=C_{S / M}(P) . \quad M<C<S$ since $C_{G / M}(S / M)=S / M$ [4, Lemma 1.2.5]. $C P$ satisfies the hypothesis of Theorem 2.5 and so $|C P|=|C / M||P|=r^{2}$ or $r^{3}$. It now follows that $|P|=|C|=r^{2}$, $C P$ is a nonabelian special 2-group of order $r^{3}$, and $\left|C_{C P}(x)\right|=r^{2}$ if $x \in C P-M$. Now $M<[S, P]<S$ and $[S, P]$ is normalized by $N$. Theorem 2.5 now implies that $|[S, P]|=r^{2}$. Since $N$ normalizes $C \cap[S, P]$ and $C \cap[S, P]>M$, we obtain, using Theorem 2.5 again, that $|C \cap[S, P]|=r^{2}$. Thus $C=[S, P]$.

Since $[P, C, S] \leqq[M, S]=1$ and $[C, S, P] \leqq[M, P]=1$, the three subgroups lemma implies $C^{\prime}=[C, C]=[S, P, C]=1$. An immediate consequence of this is that $C_{S}(x)=C_{C P}(x)=C$ for all $x \in C-M$. It follows from this that $C_{C}(x)=M$ if $x \in S-C$ or if $x \in P-M$. $Q$ centralizes any abelian 2-subgroup of $G$ which it normalizes and $C_{S}(Q)$ $=M$. Thus $Q$ does not normalize $C$. Since $N_{A}(C) \geqq S P N$, we must have $\left|A: N_{\boldsymbol{A}}(C)\right|=\left|Q: N_{Q}(C)\right|>1$. Now distinct conjugates of $C$ have only the elements of $M$ in common. This implies that $\left|Q: N_{Q}(C)\right| \leqq r$ +1 . Now $N_{Q}(C)$ is normalized by $P N, N_{Q}(C) \neq Q$, and $N_{Q}(C) \geqq C_{Q}(P)$ $=Q^{\prime}$. This implies that $N_{Q}(C)=Q^{\prime}$. Therefore $\left|Q / Q^{\prime}\right| \leqq r+1$.

If $r=4$, then $\left|Q / Q^{\prime}\right|=3$ or 5 and so $Q^{\prime}=1$. But $P / M$, which is elementary abelian of order $r$, is faithfully represented as a group of automorphisms of $Q$. Since this is impossible, we now assume $r>4$. 
Now let $H=S P$. $H$ has exponent $8, H^{\prime}=D(H)=C$, and $Z(H)=M$. We now proceed to count the number of elements of order 4 in $H$. If $x \in P-M$ and $y \in S$, then $(x y)^{2} \equiv[x, y](\bmod M)$. Thus $(x y)^{2} \in M$ if, and only if, $y M \in C_{S / M}(x)$. Let $K / M=C_{S / M}(x)$ and let $L=C_{S}(x)$. If $z \in M$, then $L=C_{S}(x z)$. Since $P / M$ is abelian, $P$ normalizes $L$. $L \cap C=C_{C}(x)=M$ and so $[L, P] \leqq L \cap C=M$. Thus $L / M \leqq C / M$ which can happen only if $L=M$. Now the mapping which maps $y$ to $[y, x]$ is a homomorphism of $K$ into $M$ with kernel $L$. Hence $|K| \leqq r^{2}$ $=|C|$. Therefore $K=C$. Thus we have shown that if $x \in P-M$ and $y \in S$, then $(x y)^{2} \in M$ if, and only if, $y \in C$.

The elements of $H$ of order dividing 4 are precisely those elements which belong to either $C P$ or $S$. Hence $H$ has exactly $|S|+|C P|-|C|$ $=2 r^{3}-r^{2}$ elements of order dividing 4 . The number of elements of order 4 in $H$ then must be $2 r^{3}-r^{2}-r$. Since all involutions are conjugate, if $y \in M^{4}$, there must be exactly $\left(2 r^{3}-r^{2}-r\right) /(r-1)$ $=2 r^{2}+r$ solutions in $H$ to $x^{2}=y$.

Now let $\chi$ be an irreducible character of $H$ whose kernel does not contain $M$, and let $K$ be the intersection of $M$ and the kernel of $\chi$. Then $|K|=r / 2, \chi(z)=\chi(1)$ if $z \in K$, and $\chi(z)=-\chi(1)$ if $z \in M-K$.

If $x \in C-M$ and $z \in M-K$, then $\chi(x z)=-\chi(x)$. But all conjugates of $x$ in $S$ belong to $M x$ and $\left|S: C_{S}(x)\right|=r$. Thus $x$ and $x z$ are conjugate in $S$. This implies that $\chi$ has the value 0 on $C-M$. We now obtain

$$
\begin{aligned}
r^{4} e_{\chi} & =\sum_{x \in H ; x^{2} \in M} \chi\left(x^{2}\right) \\
& =\chi(1)\left[r+\left(2 r^{2}+r\right)(|K|-1-(|M|-|K|))\right] \\
& =-2 r^{2} \chi(1) .
\end{aligned}
$$

Thus $\chi(1)=r^{2} / 2$.

The sum of the squares of the degrees of the irreducible characters of $H$ whose kernels do contain $M$ must be $|H / M|=r^{3}$. Therefore the number of irreducible characters of $H$ whose kernels do not con. tain $M$ is $\left(r^{4}-r^{3}\right) /\left(r^{2} / 2\right)^{2}=4(r-1) / r$. Since $r>4$, this cannot be an integer and so the theorem is proved.

\section{REFERENCES}

1. W. Feit, Characters of finite groups, Benjamin, New York, 1967. MR 36 \#2715.

2. W. Feit and J. Thompson, Solvability of groups of odd order, Pacific J. Math. 13 (1963), 775-1029. MR 29 \#3538.

3. F. Gross, On finite groups of exponent $p^{m} q^{n}$, J. Algebra 7 (1967), 238-253. MR $36 \# 273$.

4. P. Hall and G. Higman, On the p-length of p-soluble groups and reduction the- 
orems for Burnside's problem, Proc. London Math. Soc. (3) 6 (1956), 1-42. MR 17 \#344.

5. B. Huppert, Subnormale Untergruppen und p-Sylowgruppen, Acta Sci. Math. (Szeged) 22 (1961), 46-61. MR 24 \#A1310.

6. E. Shult, On finite automorphic algebras, Illinois J. Math. 13 (1969), 625-653. MR 40 \#1441.

7. J. G. Thompson, Nonsolvable finite groups all of whose local subgroups are solvable, Bull. Amer. Math. Soc. 74 (1968), 383-437. MR 37 \#6367.

University of Utah, Salt Lake City, Utah 84112 